\title{
Improved Semi-Lagrangian Stabilizing Correction Scheme for Shallow Water Equations
}

\author{
Andrei Bourchtein and Ludmila Bourchtein \\ Pelotas State University, Department of Mathematics \\ Rua Anchieta 4715 bloco K, ap.304, Pelotas 96020-250, Brazil \\ bursteinaterra.com.br
}

\begin{abstract}
Improved splitting method based on stabilizing correction scheme is used for solving shallow water equations. Recently introduced by Douglas et al. technique is applied to reduce splitting errors. Accuracy and stability analysis showed that the developed scheme allows to chose extended time steps and is more accurate as compared with standard splitting method. The results of numerical experiments confirmed that presented scheme has almost the same computational efficiency as primitive stabilizing correction method and it continues to be accurate and stable for extended time steps up to 1 hour when primitive method fails.
\end{abstract}

\section{Introduction}

For a given spatial resolution, the maximum allowable time step for systems with multiple time scales is primarily determined by the fastest processes treated explicitly in numerical scheme. In the large scale atmospheric models the main part of flow energy is in advective motions, which are much slower than the gravitational wave speeds present in the system. Since overall accuracy of numerical solution is mainly defined by approximation of slow modes, the use of explicit schemes in numerical weather prediction models is not justifiable: in such schemes the time step needed for stable integration of the high-frequency motions is more than 10 times as small as the value required by accuracy and stability conditions of the principal low-frequency modes. As a mere fact, explicit time integration has no been used in the last 20 years in the majority of atmospheric centers.

Semi-implicit time differencing is an attractive alternative for atmospheric modeling because the terms responsible for the fastest waves appear in linear form in the primitive (hydrostatic or shallow water) equations. In fact, the semi-implicit Eulerian and semi-Lagrangian methods are the most popular techniques used in numerical weather prediction and simulation of the atmospheric processes of different space and time scales: from general circulation and climate models to regional and mesoscale modeling and numerical weather prediction $[9,17]$. In the last two decades a semi-Lagrangian treatment of advection was shown to be more efficient because it allows to overcome the Courant-Friedrichs-Lewy (CFL) condition with respect to advection at the low cost of solving a set of trajectory equations. Semi-Lagrangian semi-implicit methods are used in the majority of actual research and operational atmospheric models $[6,9,11,14,15,16,17]$. 
The implicit terms of unsplit semi-implicit models require solution of $2 \mathrm{D}$ or $3 \mathrm{D}$ linear elliptic equations that is a computationally expensive task. To circumvent this problem, some time splitting techniques have been applied to atmospheric models [2, $3,5,10,12,18]$. Splitting methods allow to decouple multi-dimensional elliptic equations into a set of $1 \mathrm{D}$ problems, which are solved very efficiently by direct Gelfand-Thomas algorithm. Unfortunately, to the best of our knowledge, all reports on application of splitting techniques in atmospheric models indicate the fast grow of splitting truncation error when time step exceeds CFL advection criterion $[2,3,4,13$, $18,19]$. To reduce the splitting errors, Douglas et al. recently have proposed small modifications to splitting construction in the case of parabolic equations $[7,8]$. In this study we apply the last technique to semi-Lagrangian stabilizing correction (SLSC) scheme for shallow water equations, analyze stability and accuracy properties of the obtained scheme and present the results of numerical tests.

\section{SC Scheme for Linearized Shallow Water Equations}

Shallow water equations linearized about a state of rest can be written in the form:

$$
\partial_{t} u=f v-\partial_{x} \Phi, \partial_{t} v=-f u-\partial_{y} \Phi, \partial_{t} \Phi=-c^{2}\left(\partial_{x} u+\partial_{y} v\right) \text {. }
$$

The equations are presented in rotating reference system using Cartesian spatial coordinates $x, y$, time coordinate $t$ and common denotations for unknown functions and parameters: $u$ and $v$ are velocity components, $\Phi=g z$ is the geopotential, $g$ is the gravitational acceleration, $z$ is the height of pressure surface, $c^{2}=\Phi_{0}\left(\Phi_{0}\right.$ is a mean value of the geopotential), $f=2 \Omega \sin \varphi$ is the Coriolis parameter, $\Omega$ is the modulus of angular velocity of earth's rotation. In system (1) we assume $f=$ const .

The semi-discrete form of a single time step of considered SC scheme applied to (1) can be written as follows:

$$
\begin{gathered}
\frac{\hat{u}-u}{\tau}=f v-\partial_{x} \Phi, \frac{\hat{v}-v}{\tau}=-f u-\partial_{y} \Phi, \frac{\hat{\Phi}-\Phi}{\tau}=-c^{2}\left(\partial_{x} u+\partial_{y} v\right) ; \\
\frac{\tilde{u}-\hat{u}}{\tau}=f \frac{\tilde{v}-v}{2}-\partial_{x} \frac{\tilde{\Phi}-\Phi}{2}, \frac{\tilde{v}-\hat{v}}{\tau}=0, \frac{\tilde{\Phi}-\hat{\Phi}}{\tau}=-c^{2} \partial_{x} \frac{\tilde{u}-u}{2} ; \\
\frac{u^{\tau}-\tilde{u}}{\tau}=0, \frac{v^{\tau}-\tilde{v}}{\tau}=-f \frac{u^{\tau}-u}{2}-\partial_{y} \frac{\Phi^{\tau}-\Phi}{2}, \frac{\Phi^{\tau}-\tilde{\Phi}}{\tau}=-c^{2} \partial_{y} \frac{v^{\tau}-v}{2} .
\end{gathered}
$$

Here $\tau$ is the time step, $n$ is the step number, $\varphi(\varphi=u, v, \Phi)$ denotes the values at the "current" time level $n \tau, \hat{\varphi}, \tilde{\varphi}, \varphi^{\tau}$ denote the values at the "new" time level $(n+1) \tau$. By eliminating the intermediate functions, one can reduce system (2)-(4) to 


$$
\begin{aligned}
& \frac{u^{\tau}-u}{\tau}=f \frac{v^{\tau}+v}{2}-\partial_{x} \frac{\Phi^{\tau}+\Phi}{2}+\frac{\tau^{2}}{4}\left(u^{\tau}-u\right)+\frac{\tau f}{4} \partial_{y}\left(\Phi^{\tau}-\Phi\right)-\frac{\tau c^{2}}{4} \partial_{x y}\left(v^{\tau}-v\right), \\
& \frac{v^{\tau}-v}{\tau}=-f \frac{u^{\tau}+u}{2}-\partial_{y} \frac{\Phi^{\tau}+\Phi}{2}, \frac{\Phi^{\tau}-\Phi}{\tau}=-c^{2} \partial_{x} \frac{u^{\tau}+u}{2}-c^{2} \partial_{y} \frac{v^{\tau}+v}{2} .
\end{aligned}
$$

This implies the second order of accuracy and the following characteristic equation:

$$
(\lambda-1)\left[\left(1-\frac{\tau^{2} f^{2}}{4}\right)(\lambda-1)^{2}+\left(\frac{\tau^{2} c^{2}}{4}\left(d_{x}^{2}+d_{y}^{2}\right)+\frac{\tau^{4} c^{4}}{16} d_{x}^{2} d_{y}^{2}+\frac{\tau^{2} f^{2}}{4}\right)(\lambda+1)^{2}\right]=0
$$

Here $d_{x}, d_{y}$ are the traces of partial derivative operators and $\lambda$ is the amplification factor. Obviously, all roots of equation (7) are on the unit circle iff

$$
\tau^{2} f^{2} \leq 4
$$

which is the linear stability criterion for scheme (2)-(4) and its spatial discretizations. This is very lenient restriction because $f_{\max } \approx 1.4 \cdot 10^{-4} \mathrm{sec}^{-1}$.

\section{Improved SC Scheme for Full Shallow Water Equations}

The full shallow water equations have a form

$$
d_{t} u=f v-\partial_{x} \Phi, d_{t} v=-f u-\partial_{y} \Phi, d_{t} \Phi=-\Phi\left(\partial_{x} u+\partial_{y} v\right),
$$

where $d_{t}$ is a symbol of the substantive derivative operator.

One of the principal difficulties in applying the splitting methods to atmospheric models is the fast growth of truncation errors due to operator splitting when time step increases. Reports on computational experiments with different splitting techniques show that the overall solution errors become to be unacceptable when time step exceeds 30-40 min, although the stability criterion allows using time steps up to 1.5-2 hours or the scheme is even absolutely stable $[2,3,4,13,18,19]$. One of the recent approaches to reduce these splitting errors was proposed by Douglas et al [7, 8] for parabolic equations. Applying a similar idea to approximation of equations (9) along with efficient semi-Lagrangian treatment of advection, we obtain the following modified SLSC scheme:

$$
\begin{gathered}
\frac{\hat{u}-u}{\tau}=f v-\partial_{x} \Phi-\left[\frac{\tau f^{2}}{4}\left(u-u^{-\tau}\right)+\frac{\tau f}{4} \partial_{y}\left(\Phi-\Phi^{-\tau}\right)-\frac{\tau c^{2}}{4} \partial_{x y}\left(v-v^{-\tau}\right)\right] \\
\frac{\hat{v}-v}{\tau}=-f u-\partial_{y} \Phi, \frac{\hat{\Phi}-\Phi}{\tau}=-\Phi\left(\partial_{x} u+\partial_{y} v\right) \\
\frac{\tilde{u}-\hat{u}}{\tau}=f \frac{\tilde{v}-v}{2}-\partial_{x} \frac{\tilde{\Phi}-\Phi}{2}, \frac{\tilde{v}-\hat{v}}{\tau}=0, \frac{\tilde{\Phi}-\hat{\Phi}}{\tau}=-c^{2} \partial_{x} \frac{\tilde{u}-u}{2}
\end{gathered}
$$




$$
\frac{u^{\tau}-\tilde{u}}{\tau}=0, \frac{v^{\tau}-\tilde{v}}{\tau}=-f \frac{u^{\tau}-u}{2}-\partial_{y} \frac{\Phi^{\tau}-\Phi}{2}, \frac{\Phi^{\tau}-\tilde{\Phi}}{\tau}=-c^{2} \partial_{y} \frac{v^{\tau}-v}{2}
$$

In (11), (12) we use constant divergence coefficient, which simplifies solving the implicit equations and has no effect on practical stability and accuracy if geopotential deviations from the mean value are sufficiently small. Hereinafter all the values are located at the points of the particle trajectories calculated at each time step by the formulas $d_{t} x=u, d_{t} y=v$. Namely, $\varphi$ denotes the values at the departure points of the 2D trajectories at "current" time level $n \tau, \varphi^{-\tau}$ denotes the values at "double departure" points at the "old" time level $(n-1) \tau, \hat{\varphi}, \tilde{\varphi}, \varphi^{\tau}$ denote the values at the arrival points of the trajectories at "new" time level $(n+1) \tau$. Arrival points are chosen to be the nodes of a uniform spatial grid and the departure points are calculated by fixed point iterations (which is usually applied algorithm) with a sufficient condition of convergence given in the form [17]:

$$
\tau \leq 1 / V_{d}, \quad V_{d}=\max \left(\left|u_{x}\right|,\left|u_{y}\right|,\left|v_{x}\right|,\left|v_{y}\right|\right) .
$$

For spatial grid with meshsize $h \approx 50 \mathrm{~km}$ we have $V_{d} \approx 1.6 \cdot 10^{-4} \mathrm{~s}^{-1}$ and the maximum allowable time step is $\tau \approx 100 \mathrm{~min}$. Analysis of the linear stability of scheme (10)-(12) reveals that besides (13) there is a counterpart of (8) to be satisfied: $\tau^{2} f^{2} \leq 2$. However, the last inequality admits time step up to $\tau \approx 170 \mathrm{~min}$, which is a less restrictive than (13), and therefore it does not cause any inconvenience.

Note that if bracketed expression in (10) is omitted we obtain standard SC scheme, which is analogous to (2)-(4), but with semi-Lagrangian approximation of advective terms. This scheme has to satisfy the same condition (13).

\section{Numerical Experiments}

In Table 1 we present the results of comparison of 24-h geopotential height forecasts produced by standard (SLSC) and modified (MSLSC) schemes with "exact" forecasts obtained by using leap-frog scheme. The spatial domain $Q$ of $5000 \times 5000 \mathrm{~km}^{2}$ centered at Porto Alegre city $\left(30^{0} \mathrm{~S}, 52^{0} \mathrm{~W}\right)$ was covered by uniform spatial grid. To reveal an influence of a spatial resolution, two grids with meshsizes of $100 \mathrm{~km}$ and 50 $\mathrm{km}$ were used. The initial and boundary value conditions for geopotential and wind components on the $500 \mathrm{hPa}$ pressure surface were obtained from objective analysis and global forecasts of National Centers for Environmental Prediction (NCEP). To define the splitting errors of SLSC and MSLSC schemes as functions of time step, numerical integrations were performed for $\tau=10,20,40,60 \mathrm{~min}$. Note that computations using leap-frog scheme were carried out with time step $\tau=1 \mathrm{~min}$ (maximum allowable time step for meshsize $h=50 \mathrm{~km}$ ). Evidently, some initial error is inherent to this comparison, that is, even if $\tau$ approaches 0 , the differences 
between two forecasts do not vanish because of different spatial truncation errors for semi-Lagrangian and leap-frog methods. However, this error is quite small (less than 2 meters) and has no influence on comparison of the schemes. The results in Table 1 clearly show an advantage of modified scheme for time steps exceeding $30 \mathrm{~min}$ and comparison between $100-\mathrm{km}$ and $50-\mathrm{km}$ results confirms that principal part of truncation error consists of additional time splitting error for extended time steps.

Table 1. Root-mean-square differences between presented and reference (leap-frog) schemes. $\tau$ is the time step used for integrations (in minutes); $h$ is the meshsize of spatial grid (in kilometers); the shown numbers are root-mean-square height differences between 24-h forecasts of chosen scheme and leap-frog scheme (in meters).

\begin{tabular}{|c|c|c|c|c|c|}
\hline scheme & $h$ & $\tau=10$ & $\tau=20$ & $\tau=40$ & $\tau=60$ \\
\hline SLSC & 100 & 2.1 & 2.7 & 4.9 & 27.8 \\
\hline MSLSC & 100 & 2.1 & 2.6 & 3.5 & 4.8 \\
\hline SLSC & 50 & 2.0 & 2.6 & 4.7 & 27.3 \\
\hline MSLSC & 50 & 2.0 & 2.6 & 3.4 & 4.6 \\
\hline
\end{tabular}

In the following series of experiments, ten 24-h forecasts were calculated using leap-frog, SLSC and MSLSC schemes with 1-min, 40-min and 60-min time steps, respectively. The same $50-\mathrm{km}$ spatial grid and the same initial and boundary conditions were used. To evaluate a quality of geopotential forecastings, two common used mean scores were calculated: the root-mean-square differences between 24-h forecasts and NCEP analysis and the correlation coefficient between observed and forecast changes [1]. The results of these estimations presented in Table 2 show good level of accuracy and efficiency of MSLSC scheme. Overall values of these measures of forecast skill are quite characteristic for shallow water model [1].

Table 2. Mean objective scores of the 24-h geopotential forecasts. $\varepsilon$ and $r$ are the root-meansquare differences (in meters) and correlation coefficients (nondimensional) between analysis and $24-\mathrm{h}$ forecasting fields at the height $500 \mathrm{hPa} ; T_{C P U}$ is the CPU time for one forecast (in seconds).

\begin{tabular}{|c|c|c|c|c|}
\hline scheme & $\tau$ & $\varepsilon$ & $r$ & $T_{C P U}$ \\
\hline leap-frog & 1 & 48 & 0.83 & 41.4 \\
\hline SLSC & 20 & 46 & 0.84 & 11.1 \\
\hline SLSC & 40 & 50 & 0.82 & 5.6 \\
\hline MSLSC & 40 & 47 & 0.84 & 6.0 \\
\hline MSLSC & 60 & 48 & 0.83 & 4.1 \\
\hline
\end{tabular}

Finally, extended in time 120-h forecasts were performed with SLSC and MSLSC models using 40-min and 60-min time steps, respectively. Both integrations were stable, but forecast accuracy was lost after 72 hours of calculations. All computations were carried out on a DEC 3000 computer.

This research was supported by brazilian science foundations CNPq and FAPERGS under grants 302738/2003-7 and 02/0588.7. 


\section{References}

1. Antes R.A.: Regional models of the atmosphere in middle latitudes. Mon. Wea. Rev., 111 (1983) 1306-1335.

2. Bates J.R.: An efficient semi-Lagrangian and alternating direction implicit method for integrating the shallow water equations. Mon. Wea. Rev., 112 (1984) 2033-2047.

3. Bourchtein A.: Semi-Lagrangian semi-implicit space splitting regional baroclinic atmospheric model. Appl. Numer. Math. 41 (2002) 307-326.

4. Browning G.L., Kreiss H.-O.: Splitting methods for problems with different timescales. Mon.Wea.Rev. 122 (1994) 2614-2622.

5. Cohn S.E., Dee D., Isaacson E., Marchesin D., Zwas G.: A fully implicit scheme for the barotropic primitive equations. Mon. Wea. Rev., 113 (1985), 436-448

6. Côté J., Gravel S., Methot A., Patoine A., Roch M., Staniforth A.: The operational CMCMRB global environmental multiscale (GEM) model. Part I: Design considerations and formulation. Mon. Wea. Rev. 126 (1998) 1373-1395.

7. Douglas J., Kim S.: Improved accuracy for locally one-dimensional methods for parabolic equations. Mathematical Models and Methods in Applied Science, 11 (2001) 1563-1579.

8. Douglas J., Kim S. Lim H.: An improved alternating-direction method for a viscous wave equation, In "Current Trends in Scientific Computing", Z. Chen, R. Glowinski, and Kaitai Li, eds., Contemporary Mathematics, 329 (2003) 99-104.

9. Durran D.: Numerical Methods for Wave Equations in Geophysical Fluid Dynamics. Springer, New York (1999).

10. Kar S.K., Turco R.P., Mechoso C.R., Arakawa A.: A locally one-dimensional semiimplicit scheme for global gridpoint shallow-water models. Mon. Wea. Rev. 122 (1994) 205-222.

11. Kiehl J.T., Hack J.J., Bonan G.B., Boville B.A., Williamson D.L., Rasch P.J.: The National Center for Atmospheric Research Community Climate Model: CCM3. J. Climate 11 (1998) 1131-1149.

12. Mcdonald A.: A semi-Lagrangian and semi-implicit two time level integration scheme. Mon. Wea. Rev. 114 (1986) 824-830.

13. McDonald A., Haugen J.: A two-time-level, three-dimensional semi-Lagrangian, semiimplicit, limited-area gridpoint model of the primitive equations. Mon. Wea. Rev. 120 (1992) 2603-2621.

14. Moorthi S.: NWP Experiments with a gridpoint semi-Lagrangian semi-implicit global model at NCEP. Mon. Wea. Rev. 125 (1997) 74-98.

15. Qian J.H., Semazzi F.H.M., Scroggs J.S.: A global nonhydrostatic semi-Lagrangian atmospheric model with orography. Mon. Wea. Rev. 126 (1998) 747-771.

16. Ritchie H., Temperton C., Simmons A., Hortal M., Davies T., Dent D., Hamrud M.: Implementation of the semi-Lagrangian method in a high-resolution version of the ECMWF forecast model. Mon. Wea. Rev. 123 (1995) 489-514.

17. Staniforth A., Côté J.: Semi-Lagrangian integration schemes for atmospheric models - A review. Mon. Wea. Rev. 119 (1991) 2206-2223.

18. Tanguay M., Robert A.: Elimination of the Helmholtz equation associated with the semiimplicit scheme in a grid point model of the shallow water equations. Mon. Wea. Rev. 114 (1986) 2154-2162.

19. Yakimiw E., Robert A.: Accuracy and stability analysis of a fully implicit scheme for the shallow water equations. Mon. Wea. Rev. 114 (1986) 240-244. 